\title{
Clinical aspects of foot health and their influence on quality of life among breast cancer survivors: a case-control study
}

This article was published in the following Dove Press journal:

Cancer Management and Research

I November 2017

Number of times this article has been viewed

\author{
Patricia Palomo-López \\ David Rodríguez-Sanz ${ }^{2}$ \\ Ricardo \\ Becerro-de-Bengoa-Vallejo 3 \\ Marta Elena Losa-Iglesias ${ }^{4}$ \\ Jorge Guerrero-Martín ${ }^{5}$ \\ Cesar Calvo-Lobo ${ }^{6}$ \\ Daniel López-López ${ }^{7}$
}

'Department of Nursing, University Center of Plasencia, University of Extremadura, ${ }^{2}$ Department of Physical Therapy and Podiatry, Physical Therapy and Health Sciences, Research Group, Universidad Europea de Madrid, Madrid, ${ }^{3}$ School of Nursing, Physiotherapy and Podiatry, University Complutense of Madrid,

${ }^{4}$ Faculty of Health Sciences, University Rey Juan Carlos, ${ }^{5}$ Department of

Nursing, Faculty of Medicine, Badajoz. University of Extremadura, ${ }^{6}$ Nursing and Physical Therapy Department, Institute of Biomedicine (IBIOMED), Universidad de León, Ponferrada, León, ${ }^{7}$ Research, Health and Podiatry Unit, Department of Health Sciences, Faculty of Nursing and Podiatry, Universidade da Coruña, Spain

Correspondence: Cesar Calvo Lobo Nursing and Physical Therapy Department, Institute of Biomedicine (IBIOMED), Universidad de León,

Ponferrada, León, Spain

Tel +34 987442053

Email ccall@unileon.es
Purpose: The aim of this study was to analyze and compare foot health and general health in a sample of women divided into two groups: 1) those with breast cancer and undergoing chemotherapy treatment and 2) healthy women without breast cancer and with normalized reference values.

Methods: A case-control observational study was performed. Two-hundred women with a mean age of $51.00 \pm 8.75$ years were recruited from podiatric medicine and surgery clinics from the University of Extremadura (Plasencia, Spain) and the Hospital Infanta Cristina (Badajoz, Spain). The women were divided into case and control groups (undergoing chemotherapy treatment and healthy women, respectively). The Foot Health Status Questionnaire was used to assess foot health domain scores.

Results: Significant differences between both groups were seen for foot pain $(P=0.003)$, foot function $(P<0.001)$, physical activity $(P<0.001)$, social capacity $(P<0.001)$, and vigor $(P=0.001)$. The remaining domains (footwear, general health, and foot health) did not show significant differences between the two groups $(P \geq 0.01)$.

Conclusion: Women with breast cancer presented a lower foot health-related quality of life. Clinical aspects with emphasis on foot pain and disability were increased. Furthermore, physical activity, social capacity, and vigor were affected. Therefore, general health care and foot problem prevention for breast cancer survivors should be given more consideration.

Keywords: breast cancer, chemotherapy, foot injury, quality of life

\section{Introduction}

Quality of life (QoL) is an important health indicator in cancer patients. Cancer is viewed as the main cause of death worldwide, and it also has a significant social impact on patients. ${ }^{1}$ Among QoL studies in cancer patients, breast cancer has received the most attention. ${ }^{2}$ This disease affects the QoL of these women. ${ }^{3}$ QoL measurements during cancer patients' treatment has become an important tool over the last several years as it provides information about physical, emotional, and/or social aspects in addition to disease-associated symptoms and their treatments. ${ }^{4}$ Cancer treatments such as chemotherapy may produce side effects and symptoms that negatively affect a patient's QoL, and treatments such as mastectomies can cause lower self-esteem. ${ }^{5}$ Other aspects such as psychosocial and medical factors (age, education, emotional, labor, and/or economic situations) can influence a patient's QoL. ${ }^{4}$ Based on these aspects, foot care and its follow-up should be considered for patients suffering from common foot problems. ${ }^{3}$ 
Breast cancer is the major cancer that is prevalent among women, especially in those aged $>50$ years. ${ }^{6,7}$ The World Health Organization has stated that breast cancer is the leading cause of cancer death worldwide, with varying incidences across countries. When considering developed countries, it has been shown that cancer-related mortality rates are declining. ${ }^{6,8-10}$ In 2012, a new global study detected approximately 1.67 million newly diagnosed breast cancer cases in women. ${ }^{3}$

Breast cancer-associated chemotherapy also leads to side effects, and certain chemotherapy regimens can cause, with regard to the limbs, hand-foot syndrome or palmoplantar erythrodysesthesia, which has already been described in the scientific literature. Hand-foot syndrome is a skin reaction that occurs when a small amount of medicine gets filtered out of the capillaries (small blood vessels) and into the skin, especially into the skin of the palms of the hands and soles of the feet. After the drug has filtered out of the capillaries into the skin, it can damage the surrounding tissues. The hand-foot syndrome can be painful and may affect a patient's daily life; therefore, this study evaluated the symptoms that appeared in the feet of women with breast cancer who were undergoing chemotherapy. Thus, this could permit development of strategies to prevent these symptoms and improve the QoL of these women. Several signs and symptoms of the hand-foot syndrome have been reported in the scientific literature: 1) numbness; 2) tingling; 3) burning or itching; 4) redness (similar to sunburn); 5) inflammation; 6) discomfort; 7) tenderness; and 8) rash. ${ }^{11,12}$

Foot conditions and deformities should be taken into consideration in these patients in an attempt to provide better QoL in addition to looking after the general and podiatric well-being of these women. Therefore, the main purpose of this study was to analyze and compare both foot health and general health in two groups of women: 1) those with breast cancer undergoing chemotherapy treatment and 2) those without breast cancer (healthy women with normal references values). The second aim of this study was to observe the most frequent podiatric pathologies that may appear in the population of women with breast cancer and influence foot and general health-related QoL.

\section{Materials and methods Design and sample}

This design was a descriptive, observational, case-control study that was performed following the Strengthening the Reporting of Observational Studies in Epidemiology statement and checklist. ${ }^{13}$ Between January 2016 and May 2017, 200 patients were recruited from podiatric medicine and surgery clinics that provided treatment for foot diseases and disorders at University of Extremadura (Plasencia, Spain) and the Hospital Infanta Cristina (Badajoz, Spain). A consecutive sampling method was used to select the 200 participants. All patients provided consent and were enrolled in the study.

Inclusion criteria for the case group were women $>18$ years of age with breast cancer diagnosed by an oncologist and those who were undergoing chemotherapy treatment. Regarding the case group, patients with breast cancer had been undergoing chemotherapy treatment and were recruited during the time from the first session to the first year of treatment with chemotherapy. The control group consisted of healthy women without cancer.

Exclusion criteria for both groups were women $<18$ years of age, presence of other types of cancer, previous radiotherapy treatments, existence of foot deformities prior to undergoing chemotherapy treatment, neurological alterations, any systemic or chronic diseases, being non- or semi-independent in daily activities, and/or unable to understand instructions.

\section{Sample size calculation}

Sample size calculation was performed by means of the difference between two independent groups using the $\mathrm{G}^{*}$ Power 3.1.9.2 software and based on the foot function domain scores (mean \pm standard deviation [SD]) from the Foot Health Status Questionnaire (FHSQ) from a pilot study $(\mathrm{n}=40)$ with the two groups. ${ }^{14,15} \mathrm{~A}$ case group consisting of 20 women with breast cancer undergoing chemotherapy treatment (FHSQ foot function domain score $=67.95 \pm 27.53$ ) and a control group consisting of 20 healthy women (FHSQ foot function domain score $=54.37 \pm 29.80$ ) were used for the data analysis. Indeed, a one-tailed hypothesis, an effect size of 0.47 , an $\alpha$ error probability of 0.01 , a power (1- $\beta$ error probability) of 0.80 , and an allocation ratio (N2/N1) of 1 were used for the sample size calculations. Therefore, a total sample size of 182 women, 91 in each group, was estimated.

\section{Procedure}

A single trained examiner, who measured height and weight, examined each participant. Furthermore, body mass index (BMI) was calculated with height (meters) and weight (kilograms) using Quetelet's equation $\left(\mathrm{BMI}=\right.$ weight $/$ height $\left.^{2}\right) .{ }^{16}$ All subjects were asked to complete the FHSQ. ${ }^{15}$ The investigator remained throughout the process of completing the questionnaire to help resolve any misunderstandings. Furthermore, the podiatric examiner performed a thorough examination in order to observe the foot pathologies that appeared after chemotherapy treatment. 
Section 1 of the questionnaire consists of 13 questions reflecting foot health-related domains such as foot pain, foot function, footwear, and general foot health. This questionnaire has demonstrated a high degree of content, criterion, construct validity (Cronbach $\alpha=0.89-0.95$ ), and high retest reliability (intraclass correlation coefficient $=0.74-0.92$ ). ${ }^{15}$ Indeed, this assessment tool was shown to be the most appropriate measure of foot health-related QoL. This questionnaire presents 13 questions that assess four health domains of the feet: 1) pain; 2) function; 3) general health; and 4) footwear. Each domain has a specific number of questions (four questions with regard to pain, four with regard to function, three with regard to footwear, and two regarding general foot health). Indeed, pain and function assessments are based on physical phenomena. Footwear evaluation utilized practical aspects related to availability and shoe comfort, and the perception of general foot health was based on patients' selfassessment of the state of their feet. Several answers were permitted for each question permitted, and a Likert-type ordinal scale (words or phrases corresponding to a numeric scale) was applied to score the answers. The descriptors for this scale varied for each domain, and only one response was selected to be the most adequate and appropriate. The participant then circled this option on the Likert scale. The questionnaire did not provide a global score but rather generated an index for each domain. In order to obtain these scores, the responses were fed into a computer program that processes the data (The FHSQ, Version 1.03). After processing the data, a score ranging from 0 to 100 was possible. A score of 0 represented the worst state of foot health, while a score of 100 score was considered the best possible health status. Furthermore, the software provided graphical images to represent the outcomes. Section 2 evaluated questions that reflect four general health-related domains such as general health, physical activity, social capacity, and vigor. Questions were largely adapted from the Medical Outcomes Study 36-Item Short-Form Health, ${ }^{15}$ which has been demonstrated to be a valid measurement tool. ${ }^{17}$ The third section contained sociodemographic data such as participants' age, their medical characteristics, educational level, profession, civil status, and medical records. Furthermore, the Spanish FHSQ version showed adequate psychometric properties. ${ }^{18}$

Finally, the Spanish EuroQol Health Questionnaire 5 Dimensions, which is a widely used non-disease-specific questionnaire to evaluate health-related QoL, negatively correlated with the FHSQ for pain $(r=-0.445)$ and positively correlated with general foot health and function $(r=0.261$, $-0.579)$, confirming criterion-related validity. ${ }^{18}$

\section{Ethical considerations}

The Bioethics and Biosafety Committee at the University of Extremadura approved this study (Spain, registry number: 28/2017). All participants provided written informed consent before inclusion in the study. The ethical standards for human experimentation given in the Declaration of Helsinki (World Medical Association) and the Council of Europe Convention on Human Rights and Biomedicine in addition the Universal Declaration of the UNESCO about the Human Genome and Human Rights and other appropriate national or institutional organizations' ethical requirements were followed.

\section{Statistical analysis}

All variables were examined for normality of distribution using the Kolmogorov-Smirnov test, and data were considered to have a normal distribution if $P>0.01$. Demographic characteristics (such as age, height, weight, and BMI) and FHSQ domains (foot pain, foot function, footwear, general foot health, general health, physical activity, social capacity, and vigor) were described. Mean \pm SD for parametric data, median \pm interquartile range for nonparametric data, and maximum and minimum (range) values were used for the total sample: women with breast cancer who were undergoing chemotherapy (case group) and the healthy women (control group).

Independent Student's $t$-tests were performed to determine if differences were statistically significant when showing a normal distribution. Measurements that were not normally distributed were tested using the nonparametric Mann-Whitney $U$-test.

FHSQ Version 1.03 was used to obtain foot-related QoL scores. In all of the analyses, statistical significance was established with a $P$-value $<0.01$ and a confidence interval of $99 \%$. All analyses were performed with a commercially available software (SPSS 19.0, Chicago, IL, USA).

\section{Results}

A total sample of 200 women aged between 33 and 80 years old, mean \pm SD of $51.0 \pm 8.75$ years, completed the study. This sample was divided into 100 women with breast cancer who were undergoing chemotherapy (for the case group) and 100 women without this pathology (healthy women for the control group). The sociodemographic characteristics of the participants is given in Table 1, and the characteristics did not show any statistically significant differences for age, weight, height, or BMI $(P<0.01)$.

The results of the comparison between FHSQ scores from both groups are shown in Table 2. In section 1 of the 
Table I Comparison of sociodemographic characteristics of the total sample (women with breast cancer undergoing chemotherapy and healthy women)

\begin{tabular}{lllll}
\hline Characteristic & $\begin{array}{l}\text { Total group } \\
\mathbf{N}=\mathbf{2 0 0}\end{array}$ & $\begin{array}{l}\text { Breast cancer } \\
\mathbf{N}=100\end{array}$ & $\begin{array}{l}\text { Healthy } \\
\mathbf{N}=100\end{array}$ \\
\hline Age $($ years $)$ & $51.00 \pm 8.75^{\mathrm{a}}(33-80)$ & $53.60 \pm 9.16^{\mathrm{b}}(33-80)$ & $51.00 \pm 8.75^{\mathrm{a}}(45-55)$ & P-value \\
Weight $(\mathrm{kg})$ & $68.00 \pm 13.00^{\mathrm{a}}(40-120)$ & $68.82 \pm 12.26^{\mathrm{b}}(40-105)$ & $69.00 \pm 13.00^{\mathrm{a}}(43-120)$ & $0.013^{\mathrm{c}}$ \\
Height $(\mathrm{m})$ & $1.62 \pm 0.06^{\mathrm{b}}(1.46-1.79)$ & $1.62 \pm 0.06^{\mathrm{b}}(1.46-1.75)$ & $1.62 \pm 0.06^{\mathrm{b}}(1.50-1.79)$ & $0.704^{\mathrm{c}}$ \\
BMI $\left(\mathrm{kg} / \mathrm{m}^{2}\right)$ & $26.36 \pm 4.72^{\mathrm{b}}(17.78-43.5 \mathrm{I})$ & $26.03 \pm 4.39^{\mathrm{b}}(17.77-39.04)$ & $26.70 \pm 5.03^{\mathrm{b}}(18.75-43.5 \mathrm{I})$ & $0.5 \mathrm{I}^{\mathrm{d}}$ \\
\hline
\end{tabular}

Notes: In all the analyses, $P<0.0$ I (with a $99 \%$ confidence interval) was considered statistically significant. ${ }^{\text {Median }} \pm$ IR (range) was used. ${ }^{b}$ Mean \pm SD (range) was applied. 'Mann-Whitney U-test was utilized. 'Student's $t$-test for independent samples was performed.

Abbreviations: BMI, body mass index; IR, interquartile range; SD, standard deviation.

Table 2 Comparison of FHSQ scores of the total sample (women with breast cancer undergoing chemotherapy and healthy women)

\begin{tabular}{|c|c|c|c|c|}
\hline FHSQ domains & $\begin{array}{l}\text { Total group } \\
\mathbf{N}=\mathbf{2 0 0}\end{array}$ & $\begin{array}{l}\text { Breast cancer } \\
N=100\end{array}$ & $\begin{array}{l}\text { Healthy } \\
\mathrm{N}=100\end{array}$ & $P$-value \\
\hline Foot pain & $\begin{array}{l}66.56 \pm 39.88^{a} \\
(0-100)\end{array}$ & $\begin{array}{l}58.77 \pm 27.28^{b} \\
(0-100)\end{array}$ & $\begin{array}{l}78.00 \pm 34.00^{\mathrm{a}} \\
(0-100)\end{array}$ & $0.003^{c}$ \\
\hline Foot function & $\begin{array}{l}75.00 \pm 44.00^{\mathrm{a}} \\
(0-100)\end{array}$ & $\begin{array}{l}60.25 \pm 28.79^{b} \\
(0-100)\end{array}$ & $\begin{array}{l}88.00 \pm 31.00^{\mathrm{a}} \\
(0-100)\end{array}$ & $<\left.0.00\right|^{c}$ \\
\hline Footwear & $\begin{array}{l}37.50 \pm 47.58^{a} \\
(0-100)\end{array}$ & $\begin{array}{l}37.50 \pm 47.92^{\mathrm{a}} \\
(0-100)\end{array}$ & $\begin{array}{l}37.50 \pm 41.00^{\mathrm{a}} \\
(0-100)\end{array}$ & $0.659^{c}$ \\
\hline General foot health & $\begin{array}{l}42.75 \pm 35.00^{\mathrm{a}} \\
(0-100)\end{array}$ & $\begin{array}{l}25.00 \pm 35.00^{\mathrm{a}} \\
(0-100)\end{array}$ & $\begin{array}{l}43.00 \pm 35.00^{\mathrm{a}} \\
(0-93)\end{array}$ & $0.215^{c}$ \\
\hline General health & $\begin{array}{l}50.00 \pm 30.00^{\mathrm{a}} \\
(0-100)\end{array}$ & $\begin{array}{l}50.00 \pm 40.00^{a} \\
(0-100)\end{array}$ & $\begin{array}{l}55.00 \pm 30.00^{\mathrm{a}} \\
(0-100)\end{array}$ & $0.195^{c}$ \\
\hline Physical activity & $\begin{array}{l}61.11 \pm 44.25^{a} \\
(0-100)\end{array}$ & $\begin{array}{l}50.00 \pm 38.89^{a} \\
(0-100)\end{array}$ & $\begin{array}{l}78.00 \pm 38.00^{\mathrm{a}} \\
(17-100)\end{array}$ & $<0.00 \mathrm{I}^{\mathrm{C}}$ \\
\hline Social capacity & $\begin{array}{l}62.50 \pm 37.50^{\mathrm{a}} \\
(0-100)\end{array}$ & $\begin{array}{l}50.00 \pm 46.88^{a} \\
(0-100)\end{array}$ & $\begin{array}{l}75.00 \pm 38.00^{\mathrm{a}} \\
(0-100)\end{array}$ & $<0.00 \mathrm{I}^{\mathrm{C}}$ \\
\hline Vigor & $\begin{array}{l}38.00 \pm 31.25^{a} \\
(0-100)\end{array}$ & $\begin{array}{l}35.62 \pm 31.25^{b} \\
(0-100)\end{array}$ & $\begin{array}{l}46.43 \pm 24.4 I^{\mathrm{b}} \\
(0-100)\end{array}$ & $0.00 I^{d}$ \\
\hline
\end{tabular}

Notes: In all the analyses, $P<0.0$ ( with a $99 \%$ confidence interval) was considered statistically significant (bold data). ${ }^{\mathrm{a}}$ Median \pm IR (range) was used. ${ }^{b}$ Mean \pm SD (range) was applied. 'Mann-Whitney U-test was utilized. 'Student's $t$-test for independent samples was performed.

Abbreviations: FHSQ, Foot Health Status Questionnaire; IR, interquartile range; SD, standard deviation.

FHSQ, four specific domains regarding the foot, ie, pain, function, health, and footwear, were evaluated. FHSQ scores were higher for foot pain, foot function, and general foot health for healthy women compared to women with breast cancer. Section 2 assessed four domains of general well-being (overall health, physical function, social capacity, and vigor). Specifically, FHSQ scores were lower in all domains (general health, physical activity, social capacity, and vigor) for women undergoing chemotherapy compared to healthy women.

The women with breast cancer undergoing chemotherapy treatment showed a lower foot health-related QoL. Statistically significant differences between cancer and control groups were shown for foot pain $(P=0.003)$, foot function $(P<0001)$, physical activity $(P<0.001)$, social capacity $(P<0.001)$, and vigor $(P=0.001)$. The rest of the domains did not show any statistically significant differences between the two groups $(P \geq 0.01)$.
Considering the case group, this study also revealed that $94 \%$ ( $n=100$ ) of the participants reported having foot problems since they had started chemotherapy. Consequently, a physical examination revealed that the most frequent alterations in the feet were nail abnormalities ( $46 \%$, including nail plate color change, onychogryphosis, onychocryptosis, or onychomycosis), generalized pain (36\%), cracks and dryness (20\%), paresthesia (19\%), inflammation (10\%), varices (8\%), deformed fingers (7\%), and helomas and hardness (4\%). These pathologies were the most frequent, and some patients suffered from more than one of these conditions at the same time. Although less frequent in the feet, other pathologies observed after chemotherapy included cramps, loss of sensation, blistering, change in skin coloration, and/or loss of hair and papillomas (only in isolated cases). Furthermore, $4 \%$ of patients did not present any of these signs and symptoms after chemotherapy, and thus did not undergo any preventive measures. 


\section{Discussion}

Our aim in this study was to compare the impact on the foot health-related QoL between a group of women with breast cancer and a group of healthy women who present with normalized reference values. Foot health may be essential for the well-being and QoL of women with breast cancer. In a prior study on women's QoL and breast cancer in Arab countries, the decrease in physical function in addition to psychological and social problems was demonstrated as affecting the QoL of women with breast cancer. Nevertheless, foot health-related QoL was not evaluated. ${ }^{2}$ In Iran, a sample of 119 women with a mean age of $48.27 \pm 11.42$ years showed that chemotherapy had a negative impact on QoL and social relationship abilities, which was also a significant finding in our study. ${ }^{19} \mathrm{In}$ 2007, Mitchell demonstrated that chemotherapy imposed drastic changes in social and emotional well-being, which supported our results that demonstrated significant changes in physical activity and social ability domains. ${ }^{20}$ For Ganz et $a l,{ }^{21}$ chemotherapy was considered a concern as it may have a long-term impact on QoL. Moreover, Farrel et $\mathrm{al}^{22}$ highlighted the inability of women undergoing chemotherapy to perform tasks and reported that they practiced little or no physical activity. All of these contributions reinforced our study results.

The physical side effects of chemotherapy are well known, as is shown by chemotherapy's impact on symptoms of the hand-foot syndrome. ${ }^{23,24}$ Patients with hand-foot syndrome usually present a spectrum of symptoms ranging from burning, tingling, and cutaneous erythema to pain, edema, and ulcerations in the extremities. Injuries may interfere considerably with even the simplest of daily activities such as walking. Thus, the impact on foot health and QoL supported our study results and may help to facilitate preventive actions or treatments for these symptoms. ${ }^{23}$

Furthermore, another study reported new data about the incidence of all grades of hand-foot syndrome. In this study, $27.1 \%$ of all patients in whom the breast cancer had metastasized presented with this syndrome. In contrast to our study, the authors of this study strongly affirmed that hand-foot syndrome did not lead to a reduction in QoL and justified it by affirming that there was no available validated questionnaire for hand-foot syndrome. Moreover, these authors highlighted that the treatment stage may influence the health status of women with breast cancer. ${ }^{25}$ In addition, these same authors described the finding that only $11.9 \%$ of patients discontinued their therapies due to adverse events, which may have been secondary to metastatic breast cancer and no other type of cancer. Compared with other side effects, physicians may attach greater importance to dermatological or podiatric signs and symptoms in the feet than patients actually do.

Our study agrees with a study performed in 2015 in which recognition of the first signs and symptoms of handfoot syndrome was found accelerate the use of management strategies. ${ }^{26}$ In addition, hand-foot syndrome lesions show a range of signs and symptoms from numbness to painful erosions. Therefore, this may have a significant impact on the QoL in affected patients. In agreement with our study, prior studies have tried to find ways to alleviate and prevent this syndrome. Thus, prior studies about preventive strategies have described the use of topically applied ointments with high content of radical protection factor or topical applications of antioxidants with high protection factors. Both of these have been demonstrated to be effective prevention strategies in these patients. ${ }^{12}$

The results of our study suggest that women with breast cancer undergoing chemotherapy present significantly different scores on the dimensions related to foot pain, foot function, physical activity, social ability, and vigor compared to healthy women. Therefore, it seems important to note that podiatric and medical care may be useful in women with breast cancer who have undergone or are undergoing chemotherapy in order to improve their QoL. Comparison of the impact of these results with other breast cancer and chemotherapy studies may be difficult due to differences in criteria and methodological variations. We have not been able to find many studies in the literature that used the FHSQ to relate QoL and foot health in this group of patients.

This study had important limitations that should be acknowledged. A larger and more diverse sample with individuals from various countries should be recruited in order to strengthen the results from the present study regarding chemotherapy and QoL. Also, additional evaluations of women with breast cancer again at 18 and 24 months after initiation of treatment would be helpful in order to consider the longer term effects of chemotherapy on general and foot health. Finally, women with breast cancer undergoing chemotherapy treatment were recruited, although data regarding the type of drugs or chemotherapy regimens were not collected. These important considerations should be addressed in future research studies in order to evaluate the relationship between foot symptoms and certain chemotherapy drugs. Furthermore, women with breast cancer undergoing no chemotherapy treatment (positive controls) were not recruited. Future studies should compare the foot-related QoL between breast cancer patients undergoing and not undergoing chemotherapy treatment. 


\section{Conclusion}

In conclusion, measurable differences of association between women with breast cancer undergoing chemotherapy and those without breast cancer showed an impaired foot healthrelated QoL in breast cancer patients undergoing chemotherapy. Thus, this study provided evidence that chemotherapy and breast cancer may be associated with lower scores for dimensions related to foot pain, foot function, physical activity, social ability, and vigor. These items are indicative of a deterioration of specific foot-related QoL. Therefore, proper foot care and prevention may be extremely important to prevent the onset or development of lesions, pain, infections, and/or deformities throughout the chemotherapy process. Based on our research, a more exhaustive interventional study may be necessary to prevent or mitigate the negative effects after chemotherapy in this type of cancer, thereby improving foot and general QoL in these patients.

\section{Acknowledgments}

We thank the patients of Clinic of Podiatric Medicine and Surgery, University of Extremadura (Plasencia), University Hospital Infanta Cristina of Badajoz (Extremadura) y Oncology Association of Extremadura, Spain. The authors did not receive any financial assistance from or have any personal relationships with other people or organizations that could inappropriately influence (bias) their work.

\section{Author contributions}

All authors: concept, design, analyses, interpretation of data, drafting of manuscript, or revising it critically for important intellectual content.

\section{Disclosure}

The authors report no conflicts of interest in this work.

\section{References}

1. Wright EP, Kiely MA, Lynch P, Cull A, Selby PJ. Social problems in oncology. Br J Cancer. 2002;87(10):1099-1104.

2. Haddou Rahou B, El Rhazi K, Ouasmani F, et al. Quality of life in Arab women with breast cancer: a review of the literature. Health Qual Life Outcomes. 2016;14(1):64.

3. Ferlay J, Soerjomataram I, Dikshit R, et al. Cancer incidence and mortality worldwide: Sources, methods and major patterns in GLOBOCAN 2012. Int J Cancer. 2015;136(5):E359-E386.

4. Lehto US, Ojanen M, Kellokumpu-Lehtinen P. Predictors of quality of life in newly diagnosed melanoma and breast cancer patients. Ann Oncol Off J Eur Soc Med Oncol. 2005;16(5):805-816.

5. Montazeri A, Vahdaninia M, Harirchi I, Ebrahimi M, Khaleghi F, Jarvandi S. Quality of life in patients with breast cancer before and after diagnosis: an eighteen months follow-up study. BMC Cancer. 2008;8(1):330.
6. Key TJ, Verkasalo PK, Banks E. Epidemiology of breast cancer. Lancet Oncol. 2001;2(3):133-140.

7. Piñeros M, Ferlay J, Murillo R. Cancer incidence estimates at the national and district levels in Colombia. Salud Publica Mex. 48(6):455465. Available from: http://www.ncbi.nlm.nih.gov/pubmed/17326341. Accessed September 9, 2017.

8. Murray CJ, Lopez AD. Mortality by cause for eight regions of the world: Global Burden of Disease Study. Lancet. 1997;349(9061):1269-1276.

9. Boyle P. Breast cancer control: signs of progress, but more work required. Breast. 2005;14(6):429-438.

10. Veronesi U, Goldhirsch A, Boyle P, Orecchia R, Viale G. Breast cancer. Discov Med. 2005;5(27):271-277. Available from: http://www.ncbi.nlm. nih.gov/pubmed/20704887. Accessed September 9, 2017.

11. Palaniappan M, Srinivasamurthy S, Dubashi B, Chandrasekaran A. Anticancer drug induced palmar plantar erythrodysesthesia. J Clin Diagn Res. 2014;8(10):HC01-HC03.

12. Jung S, Sehouli J, Chekerov R, et al. Prevention of palmoplantar erythrodysesthesia in patients treated with pegylated liposomal doxorubicin (Caelyx $\left.{ }^{\circledR}\right)$. Support Care Cancer. Epub 2017 June 26.

13. Vandenbroucke JP, von Elm E, Altman DG, et al. Strengthening the Reporting of Observational Studies in Epidemiology (STROBE): explanation and elaboration. Int J Surg. 2014;12(12):1500-1524.

14. Faul F, Erdfelder E, Lang AG, Buchner A. G*Power 3: a flexible statistical power analysis program for the social, behavioral, and biomedical sciences. Behav Res Methods. 2007;39(2):175-191. Available from: http:// www.ncbi.nlm.nih.gov/pubmed/17695343. Accessed June 20, 2017.

15. Bennett PJ, Patterson C, Wearing S, Baglioni T. Development and validation of a questionnaire designed to measure foot-health status. J Am Podiatr Med Assoc. 1998;88(9):419-428.

16. Garrow JS, Webster J. Quetelet's index (W/H2) as a measure of fatness. Int J Obes. 1985;9(2):147-153. Available from: http://www.ncbi.nlm. nih.gov/pubmed/4030199. Accessed June 22, 2017.

17. Landorf KB, Radford JA. Minimal important difference: values for the foot health status questionnaire, foot function index and visual analogue scale. Foot. 2008;18(1):15-19.

18. Cuesta-Vargas A, Bennett P, Jimenez-Cebrian AM, Labajos-Manzanares MT. The psychometric properties of the Spanish version of the Foot Health Status Questionnaire. Qual Life Res. 2013;22(7):1739-1743.

19. Safaee A, Moghimi-Dehkordi B, Zeighami B, Tabatabaee H, Pourhoseingholi $\mathrm{M}$. Predictors of quality of life in breast cancer patients under chemotherapy. Indian J Cancer. 45(3):107-111. Available from: http:// www.ncbi.nlm.nih.gov/pubmed/19018114. Accessed September 9, 2017.

20. Mitchell T. The social and emotional toll of chemotherapy - patients' perspectives. Eur J Cancer Care (Engl). 2007;16(1):39-47.

21. Ganz PA, Desmond KA, Leedham B, Rowland JH, Meyerowitz BE, Belin TR. Quality of life in long-term, disease-free survivors of breast cancer: a follow-up study. J Natl Cancer Inst. 2002;94(1):39-49. Available from: http://www.ncbi.nlm.nih.gov/pubmed/11773281. Accessed September 9, 2017.

22. Farrell C, Heaven C, Beaver K, Maguire P. Identifying the concerns of women undergoing chemotherapy. Patient Educ Couns. 2005;56(1):72-77.

23. Nam SH, Choi HJ, Kang WD, et al. Development and Validation of the Korean Version of Hand-Foot Skin Reaction and Quality of Life Questionnaire (HF-QoL-K). J Korean Med Sci. 2016;31(12):1969-1975.

24. Degen A, Alter M, Schenck F, et al. The hand-foot-syndrome associated with medical tumor therapy - classification and management. J Dtsch Dermatol Ges. 2010;8(9):652-661.

25. Müller V, Fuxius S, Steffens CC, et al. Quality of life under capecitabine (Xeloda ${ }^{\circledR}$ ) in patients with metastatic breast cancer: data from a german non-interventional surveillance study. Oncol Res Treat. 2014;37(12):748-755.

26. Anderson RT, Keating KN, Doll HA, Camacho F. The hand-foot skin reaction and quality of life questionnaire: an assessment tool for oncology. Oncologist. 2015;20(7):831-838. 
Cancer Management and Research

\section{Publish your work in this journal}

Cancer Management and Research is an international, peer-reviewed open access journal focusing on cancer research and the optimal use of preventative and integrated treatment interventions to achieve improved outcomes, enhanced survival and quality of life for the cancer patient.

The manuscript management system is completely online and includes

Submit your manuscript here: https://www.dovepress.com/cancer-management-and-research-journal 